\title{
Ingestion of herbal medication during pregnancy and adverse perinatal outcomes
}

\author{
L S Kekana, MB ChB, FCOG, MMed; M H Sebitloane, MB ChB, FCOG, MMed, PhD
}

Discipline of Obstetrics and Gynaecology, School of Clinical Medicine, College of Health Sciences, University of KwaZulu-Natal, Durban, South Africa

Corresponding author: S H Sebitloane (Sebitloanem@ukzn.ac.za)

Background. Pregnant women in South Africa (SA) traditionally use herbal medicines even though the potential risk or benefit is not fully elucidated.

Objectives. To determine the prevalence of the use of herbal medicines in pregnant women in our setting, as well as explore the reasons for use.

Methods. This cross-sectional study was conducted at King Edward VIII Hospital, from 1 September 2014 to 31 December 2014. Women were interviewed using a structured questionnaire during the post-delivery period, irrespective of outcome. The questionnaires enquired about women's demographic data, social and previous obstetric history and herbal medication use during current pregnancy.

Results. Two hundred and ninety-nine women were interviewed. The prevalence of herbal medication use was $33.7 \%(n=101)$, mainly via the oral route. Fifty-eight (57.4\%) of these women used herbal medication throughout their pregnancy. Reasons given for herbal ingestion included general well-being, or to make labour easier or come sooner. There was a high rate of caesarean delivery among pregnant women who used herbal medication compared with those who did not $(79.2 \%$ v. $52.8 \%$; $p=0.001)$. One hundred and eighteen women had meconium-stained liquor; $59 \%$ of the herbal medication users compared with $29.6 \%$ of the non-users $(p<0.001)$ comprised this group.The perinatal mortality rate was also higher among users $(p<0.04)$. There were no maternal deaths.

Conclusion. Herbal medicine was used by a third of black South African pregnant women in this study and was associated with significant adverse obstetric and perinatal outcomes.

S Afr J Obstet Gynaecol 2020;26(2):71-75. https://doi.org/10.7196/SAJOG.2020.v26i2.1615

Herbal preparations have historically been used for general maintenance of good health, as well as prevention and management of a wide range of disorders. Herbal medicines are preparations derived from naturally occurring plants (seeds, roots, bark, stems, leaves, fruits and flowers) for their medicinal or preventive properties. Globally, the use of herbal medicines is rising in a variety of patient groups, including pregnant women, and among healthy people. It is estimated that $80 \%$ of the world's population use herbal medicine for health management. ${ }^{[1]}$ Interestingly, herbal medicine use in pregnancy has a wide range of prevalence of between 7 and $96 \%,{ }^{[2]}$ with higher prevalence in developing countries. Since ancient times, herbal medicines have been used worldwide to treat pregnancy-related illnesses, to promote healthy pregnancies and for overall well-being. They have also been recommended by some midwives to facilitate labour. ${ }^{[3,4]}$ It is estimated that $4-62 \%$ of pregnant women continue to take herbal medicines during their pregnancy despite lack of safety and efficacy data regarding their use during pregnancy. $\cdot^{[4-6]}$

Herbal medicines are seen as natural, safe, complementary alternatives to existing conventional medicines. However, some plants have toxic bioactive components that have the potential to elicit adverse reactions similar to synthetic drugs which stimulate uterine muscle and are therefore not recommended for use during pregnancy. ${ }^{[7,8]}$ Plants may be contaminated with pesticides and heavy metals which may negatively impact pregnancy. It has been shown that consumption of herbal medicines contaminated by lead can result in preterm babies with elevated levels of lead in the blood. ${ }^{[9]}$ Lead poisoning in pregnant women has been associated with the use of Ayurvedic medicines from India. ${ }^{[10]}$ In a preclinical study, twenty of the Chinese herbal medicines which are prescribed during pregnancy were administered to pregnant mice. ${ }^{[1]}$ Clinical doses were used at various gestational stages. The study showed adverse pregnancy outcomes, especially in early pregnancy, including maternal and perinatal mortality. In addition, maternal and postnatal weight gain was decreased significantly. Fetal resorption and skeletal malformations were increased significantly. ${ }^{[1]} \mathrm{A}$ prospective study in 2006 by Chuang et al. ${ }^{[12]}$ showed that taking herbal medication during the first trimester was associated with an increased risk of congenital malformation, especially of the nervous system, muscle, eyes and connective tissue.

In South Africa (SA), plants such as Clivia miniata, Agapanthus africanus and Typha capensis have been used in traditional herbal remedies for pregnant mothers. ${ }^{[13]}$ Locally, there is a belief among many urban and rural African communities that ingestion of herbal medication by a pregnant woman protects her and her unborn from harm, as well as preserving reproductive health. ${ }^{[14]} \mathrm{A}$ herbal remedy known as isihlambezo (a popular Zulu concoction made from varying constituents) is believed to assist in the delivery of a healthy baby and is made by extracting the mineral salts from plant roots, bark and wood. ${ }^{[15]}$ Varga and Veale ${ }^{[14]}$ reported in 1997 that nearly $90 \%$ of mothers felt that isihlambezo was a helpful part of self-care during pregnancy. Furthermore, it was used to treat various 
pregnancy ailments, including high blood pressure, oedema and indigestion, among others. A previous study in our setting more than 10 years ago found that $55 \%$ of patients who used this herbal medication had a higher frequency of meconium-stained liquor (MSL) and an increased number of caesarean sections. ${ }^{[13]}$ Therefore, this study aimed to investigate current use of herbal medications during pregnancy, and their possible association with MSL, stillbirth and other obstetric parameters.

\section{Methods}

This cross-sectional study was conducted at King Edward VIII Hospital, a tertiary referral hospital, in Durban, KwaZulu-Natal Province, SA, from 1 September to 31 December 2014. The study was approved by the Biomedical Research Ethics Committee (ref. no. BE: 376/13).

During the study period postpartum mothers were conveniently sampled before discharge. Following verbal informed consent, a face-to-face interview was conducted using a structured questionnaire. We included women of all ages and parity, regardless of their antenatal course, the mode of delivery or the outcome of the pregnancy. Exclusion criteria were delivery of a fetus with a birthweight $<500 \mathrm{~g}$ and gestation age $<24$ weeks. Two hundred and ninety-nine women were recruited consecutively. Details of the pregnancy and obstetric data/outcomes were extracted from the maternal records.

\section{Statistical analysis}

Data were analysed using SPSS version 22.0 (IBM Corp; USA) and presented as mean (standard deviation (SD)), frequency and percentages. Chi-square and the distribution proportion tests ( $z$-test) were used to compare herbal medication users and nonusers. A $p$-value $<0.05$ was considered statistically significant.

\section{Results}

The demographic and obstetric data of the women interviewed are shown in Table 1. Of these, 101 (33.7\%) women reported taking herbal medication during the course of the pregnancy. The mean (SD) age of the group was 26.6 (6.8) years (range 15 - 42 years) (Table 1) with herbal medication users (referred to as users) being older, mean (SD) age was 27.9 (7.0) compared with $25.9(6.6)$ years in the non-herbal group (non-users) $(n=198 ; 66.3 \%)(p=0.02)$. The lowest prevalence of herbal medication use was in women aged $\leq 20$ and $\geq 41$ years. Other demographic characteristics are shown in Table 1 , showing higher parity among users, who were more likely to be married ( $24.8 \%$ compared with $8.5 \%$ of the non-users; $p=0.001$ ), and were also less likely to have completed high school education $(p=0.001)$.

The laboratory parameters of haemoglobin, Rhesus factor and syphilis serology were comparable between the users and non-users. Among the users, 51.5\% $(n=52 / 101)$ were HIV-infected, compared with $31.3 \%(n=62 / 198)$ among non-users $(p=0.001)$.

\section{Details of herbal medication used}

Among the 101 users, the oral route was most common $(97.0 \%$ of users) with most indicating an amount of half a cup at a time (ranged from a teaspoon to a full cup). Four women used the rectal route. Fifty-eight $(57.4 \%)$ used herbal medication throughout their pregnancy, with $7.8 \%, 12.9 \%$ and $11.9 \%$ using the herbal medication exclusively in the first, second and third trimesters, respectively. The use of herbal remedies was recommended by family in most cases $(72.3 \%)$, and reasons for consumption were a combination of factors, including for general well-being (68.3\%), for labour to come sooner $(12.9 \%)$ and for the delivery to be smooth (18.8\%). Fiftyeight $(57.4 \%)$ of the users had received advice to discontinue the medication from a healthcare worker.

Table 1. Demographic and obstetric data characteristics of the study population $(N=299)$

\begin{tabular}{|c|c|c|c|}
\hline \multirow[b]{2}{*}{ Variable } & \multicolumn{2}{|c|}{ Herbal medication ingestion } & \multirow[b]{2}{*}{$p$-value } \\
\hline & Yes $(n=101), n(\%)^{*}$ & No $(n=198), n(\%)^{*}$ & \\
\hline Age (years), mean (SD) & $27.9(7.0)$ & $25.9(6.6)$ & 0.001 \\
\hline Median (range) & $27(16-42)$ & $25(15-42)$ & \\
\hline \multicolumn{4}{|l|}{ Age groups (years) } \\
\hline$\leq 20$ & $18(17.8)$ & $57(28.8 \%)$ & 0.03 \\
\hline $21-30$ & $47(46.5)$ & $86(43.4 \%)$ & 0.5 \\
\hline $31-40$ & $32(31.7)$ & $54(27.2 \%)$ & 0.4 \\
\hline$\geq 41$ & $4(4.0)$ & $1(0.5 \%)$ & 0.04 \\
\hline Parity, mean (SD) & $1.3(1.3)$ & $1.0(1.0)$ & 0.001 \\
\hline Median (range) & $1(1-6)$ & $1(1-4)$ & \\
\hline Gravidity, mean (SD) & $2.4(1.3)$ & $2.1(1.1)$ & 0.001 \\
\hline Median (range) & $2(1-7)$ & $2(1-5)$ & \\
\hline \multicolumn{4}{|l|}{ Education } \\
\hline No matric & $78(77.2)$ & $81(40.9)$ & \\
\hline GA at booking (weeks), mean (SD) & $20.9(7.5)$ & $19.2(5.5)$ & 0.001 \\
\hline Antenatal care (weeks), mean (SD) & $6.48(2.6)$ & $6.29(2.1)$ & 0.45 \\
\hline Median (range) & $6(2-12)$ & $6(1-16)$ & \\
\hline \multicolumn{4}{|l|}{ Marital status } \\
\hline Single & $74(73.3)$ & $181(91.4)$ & 0.001 \\
\hline Married & $25(24.8)$ & $17(8.5)$ & 0.001 \\
\hline Divorced & $2(1.9)$ & 0 & 0.04 \\
\hline
\end{tabular}




\section{Obstetric profile}

The mean gestational age (SD) at delivery was 36.9 (3.9) weeks for users compared with 37.8 (2.9) for non-users $(p=0.001)$ (Table 2) although the actual prematurity rate ( $<37$ weeks) did not differ. Labour was induced in $13(12.9 \%)$ women in the users group compared with $44(22.2 \%)$ in women who were non-users $(p=0.05)$. A total of $21 / 101(20.8 \%)$ users had normal vaginal delivery, whereas vaginal delivery was achieved in $94 / 198(47.4 \%)$ non-users $(p=0.001$ ) (Table $2)$, indicating a higher caesarean section rate among users, $(79.2 \% \mathrm{v}$. $52.5 \% ; p=0.001$ ). For the whole group the median duration for the first stage of labour (SD) was 8.9 (3.9) hours (range: 1 - 22), but significantly shorter for users 7.5 (3.6) compared with 9.7 (3.9) hours in non-users ( $p=0.001)$. Similarly, the second stage of labour (SD) was shorter, 10.6 (6.4) minutes compared with 13.5 (9.2) minutes in non-users ( $p=0.03$ ).

The indications for caesarean section were similar for both groups; however, MSL was significantly higher in the users group. MSL grade II or III occurred in $51.5 \%$ of users and in $17.6 \%$ of non-users $(p=0.001)$. Suboptimal cardiotocography (CTG) was also significantly more common in users, 51/101 (50.5\%) compared with
$71 / 198(35.8 \%)$ in non-users ( $p=0.01)$. Interestingly, none of the users had caesarean section for failed induction of labour (Table 2).

\section{Perinatal outcomes}

The mean (SD) birthweight was comparable (2.83 (0.6) v. 2.96 (0.6) kg; $p=0.09$ ) (Table 3).The mean (SD) Apgar scores at 1 minute were 7.04 (1.5) in users compared with $7.6(1.03)$ in non-users $(p=0.001)$. However, these were similar at 5 minutes. Meconium exposure, meconium suctioning and admission to nursery were all significantly associated with herbal medication use. Twelve perinatal deaths occurred among the users ( 9 stillbirths and 3 neonatal deaths), a perinatal mortality ratio (PNMR) of 118/1 000 live births, compared with 11 perinatal deaths (7 stillbirths and 4 neonatal deaths) in the non-users group $(\mathrm{PNMR}=55.3 / 1000$ live births $)(p<0.001)$ (Table 3$)$.

\section{Maternal comorbidities and outcome}

There was no difference in the maternal conditions in terms of preeclampsia and obstetric haemorrhage between users and non-users (Table 4).

Table 2. Obstetric and labour details

\begin{tabular}{|c|c|c|c|}
\hline \multirow[b]{2}{*}{ Variable } & \multicolumn{2}{|c|}{ Herbal medication ingestion } & \multirow[b]{2}{*}{$p$-value } \\
\hline & Yes $(n=101), n(\%)^{*}$ & No $(n=198), n(\%)^{*}$ & \\
\hline GA at delivery (wks), mean (SD) & $36.9(3.9)$ & $37.8(2.9)$ & 0.001 \\
\hline \multicolumn{4}{|l|}{ Liquor, $n(\%)$} \\
\hline Clear & $41(40.6)$ & $139(70.2)$ & 0.001 \\
\hline MSL grade I & $8(7.9)$ & $24(12.1)$ & 0.2 \\
\hline MSL grades II and III & $52(51.5)$ & $35(17.7)$ & 0.001 \\
\hline \multicolumn{4}{|l|}{ Mode of delivery, $n(\%)$} \\
\hline NVD & $21(20.8)$ & $94(47.4)$ & 0.001 \\
\hline CS & $80(79.2)$ & $104(52.5)$ & 0.001 \\
\hline Induction of labour, $n(\%)$ & $13(12.9)$ & $44(22.2)$ & 0.05 \\
\hline \multicolumn{4}{|c|}{ Fetal well-being prior to delivery, $n$ (\%) } \\
\hline CTG good & $27(26.7)$ & $109(55.1)$ & 0.001 \\
\hline Suboptimal CTG & $51(50.5)$ & $71(35.9)$ & 0.01 \\
\hline Not documented & $23(22.8)$ & $18(9.1)$ & 0.001 \\
\hline
\end{tabular}

Table 3. Foetal outcome details

\begin{tabular}{|c|c|c|c|}
\hline \multirow[b]{2}{*}{ Variable } & \multicolumn{2}{|c|}{ Herbal medication ingestion } & \multirow[b]{2}{*}{$p$-value } \\
\hline & Yes $(n=101), n(\%)^{*}$ & No $(n=198), n(\%)^{*}$ & \\
\hline \multicolumn{4}{|l|}{ Apgar scores, mean (SD) } \\
\hline $1 \mathrm{~min}$ & $7.04(1.5)$ & $7.6(1.03)$ & 0.001 \\
\hline $5 \mathrm{~min}$ & $8.3(1.2)$ & $8.7(1.1)$ & 0.41 \\
\hline \multicolumn{4}{|l|}{ Fetal outcome } \\
\hline Alive & $92(91.1)$ & $191(96.5)$ & 0.05 \\
\hline Neonatal deaths & $3(3.0)$ & $4(2.0)$ & 0.6 \\
\hline Meconium suction & $34(33.7)$ & $27(13.6)$ & 0.001 \\
\hline Neonatal complication & $33(32.7)$ & $44(22.2)$ & 0.04 \\
\hline \multicolumn{4}{|l|}{ Type of complication } \\
\hline Prematurity & $11(10.9)$ & $24(12.1)$ & 0.7 \\
\hline Birth asphyxia & $9(8.9)$ & $5(2.5)$ & 0.01 \\
\hline Congenital abnormality & $3(2.9)$ & $3(1.5)$ & 0.3 \\
\hline Meconium aspiration & $6(5.9)$ & $6(3.0)$ & 0.2 \\
\hline Birthweight (kg), mean (SD) & $2.83(0.6)$ & $2.96(0.60)$ & 0.09 \\
\hline
\end{tabular}


RESEARCH

Table 4. Maternal comorbidities and complications

\begin{tabular}{|c|c|c|c|}
\hline \multirow[b]{2}{*}{ Variable } & \multicolumn{2}{|c|}{ Herbal medication ingestion } & \multirow[b]{2}{*}{$p$-value } \\
\hline & Yes $(n=101), n(\%)$ & No $(n=198), n(\%)$ & \\
\hline \multicolumn{4}{|l|}{ Maternal comorbidities } \\
\hline Pre-eclamptic toxaemia & $4(34.0)$ & $18(9.0)$ & 0.1 \\
\hline Eclampsia & $1(01.0)$ & $1(0.5)$ & 0.6 \\
\hline Antepartum haemorrhage & $3(3.0)$ & $1(0.5)$ & 0.07 \\
\hline \multicolumn{4}{|l|}{ Maternal complications } \\
\hline Postpartum haemorrhage & $1(1.0)$ & $6(3.0)$ & 0.2 \\
\hline
\end{tabular}

\section{Discussion}

The prevalence of herbal medication use among pregnant women in our study was $33.7 \%$, lower than a previous study which found $55 \%$ in the same setting. ${ }^{[13]}$ It is possible that women may not have been willing to divulge the information, especially if they had an adverse outcome, and also because more than half confessed having been advised against the use of the medication at some point during the pregnancy. Some women may have forgotten if they had taken the medicine; however, this is unlikely as most women hold strong beliefs either for or against use. Some rural environments may be less intimidating possibly because the use of herbal medicines is the norm in the community setting. Under-reporting has been cited as a possibility in another urban study in SA (Baragwanath Hospital in Soweto). ${ }^{[16]}$ Other countries have reported a higher rate of use, $57.8 \%$ in England, ${ }^{[17]}$ and $51.4 \%$ in Malaysia. ${ }^{[18]}$

Family dynamics influencing the use of herbal medications were demonstrated in this study, in that users were more likely to be older, married and of higher parity, though the majority in both groups were not married. Sooi and Keng ${ }^{[19]}$ documented that more pregnant women using herbal medication (86.4\%) were likely to be $21-40$ years of age.

The majority of mothers (57.4\%) ingested herbal medication throughout the pregnancy. A former study in the same setting showed that the majority of women ingested herbal medication in their third trimester, and were likely to have used herbal medication $<12$ hours prior to hospital admission. ${ }^{[13]}$ Several studies have also reported that $53.3 \%$ of mothers ingested herbal medication during the first trimester ${ }^{[22]}$ and $79.6 \%$ in the third trimester. ${ }^{[18]}$ The high incidence of herbal consumption in the first trimester is of concern. At this stage, fetal development is most vulnerable and exposure to any type of medication may result in adverse outcomes. Herbal medication ingestion during the first trimester is associated with increased risk of congenital malformation. ${ }^{[12]}$ In our study there were three cases of congenital abnormalities in each group. The minimal use in the first trimester (and therefore no significant impact on congenital abnormalities) may also demonstrate that pregnancies are often hidden in the first months in this largely unmarried population.

Preclinical and clinical studies on herbal medicines use during pregnancy have demonstrated increased neonatal morbidity and mortality. ${ }^{[11,20,21]}$ Our study confirms previous findings of adverse outcomes associated with herbal medication use, with increased perinatal mortality, increased caesarean section, and poor indicators of fetal well-being. These included passage of MSL, more suboptimal CTGs, and more infants with aspiration and needing suctioning, as well as admissions to nursery. Mabina et al. ${ }^{[13]}$ reported no stillbirths or neonatal deaths but 5 babies developed neonatal asphyxia, 3 of whom were from non-herbal users. ${ }^{[13]}$ In another study, the use of herbal medication during pregnancy was associated with an increased risk of prematurity; ${ }^{[22]}$ this was not shown in our study although mean gestational age at delivery was earlier in the user group. Herbal medicines use during the second/third trimesters may lead to intrauterine growth retardation, ${ }^{[23]}$ fetal distress, ${ }^{[13]}$ fetal hypoxia ${ }^{[14]}$ and intrauterine death. In this study, despite the majority of women $(57.4 \%)$ using the medication throughout the pregnancy, there was no significant difference in birthweight between the two groups, although gestational age at delivery was different (Tables 2 and 3). A study conducted in Norway found that mothers who ingested iron-rich traditional herbs had babies of increased weight. ${ }^{[12,14}$ This variance in weight could be due to the type of herbal medication consumed and bioactive compounds present.

Various reasons exist for herbal medication ingestion during pregnancy. The primary reason is to improve the mother's and baby's health. ${ }^{[5,24]}$ A study in Nigeria ${ }^{[25]}$ reported that mothers ingested herbal medication because they considered the herbs to be harmless, easily accessible and affordable. Rahman et al. ${ }^{[18]}$ reported that common indications for using herbal medicines during pregnancy were to facilitate labour (89.8\%), promote baby's physical health and intelligence $(8.3 \%)$, prevent retained placenta $(0.9 \%)$ and prevent abortion $(0.9 \%)$. In our study, the reasons for herbal medication consumption were the mothers' general well-being, labour to come sooner and delivery to be easier. Evidence of the uterotonic effects of isihlambezo seems convincing in that the labour came earlier for the users, with none requiring induction of labour (commonly performed for postdatism). Additionally, both the duration of the first and second stages of labour were significantly shorter among the users. In another study more women who used herbal medication had antepartum haemorrhage from abruptio placentae. ${ }^{[13]}$ Our study showed increased passage of MSL and a higher rate of suboptimal CTGs among users. Further pharmacological studies may provide more information on whether a lower dosage would be less harmful and therefore avoid such adverse outcomes.

Another finding of our study was a higher HIV infection rate (51.5\%) among users, compared with non-users (31.3\%). The latter figure is in keeping with the national antenatal HIV seroprevalence survey ${ }^{[26]}$ This is a confounding factor, and it may be that HIVinfected women are already feeling unwell, or alternatively looking for anything that promises better health for them and their unborn babies. However, in this context, further community education should be embarked upon to alert women to the potential hepatotoxic and nephrotoxic side-effects of both antiretroviral agents and herbal medication, which may be additive.

\section{Study limitations}

Recall bias is possible, especially on whether herbal medication was used earlier in pregnancy. Reluctance to disclose use may have led to 
underestimation of the actual number who used herbal medication. Complications of pregnancy prior to 24 weeks and birthweights $<500 \mathrm{~g}$ were not assessed as these were exclusion criteria of the study.

\section{Conclusions}

The findings of this study show that one-third of an urban population of pregnant women in KwaZulu-Natal Province, SA, continue to take herbal medications, with the belief that the medication aids towards better pregnancy and a good outcome. The findings of higher adverse pregnancy outcomes, such as fetal compromise and a subsequent increased rate of caesarean section among users, are significant enough to cause alarm and require caution. Because herbal medication use is entrenched in local culture, this calls for urgent community engagement and education, paralleled with scientific exploration of the actual pharmacokinetics of the agents used.

\section{Declaration. None.}

Acknowledgements. We would like to thank Dr C Tiloke for assistance with the manuscript preparation.

Author contributions. LSK assisted in protocol development, collection and analysis of data, and manuscript write-up. MHS conceived the idea, supervised the project, assisted in interpretation of data and the manuscript preparation.

Funding. None.

Conflicts of interest. None

1. Sekhar MS, Aneesh TP, Varghese KJ, Vasudaven DT, Revikumar KG. Herbalism: A phenomenon of new age in medicine. Internet J Pharmacol 2008;6(1):1-12

2. Wambebe C. Regulatory framework for local production of herbal medicines in Africa. Bol Latinoam Caribe Plant Med Aromat 2009;8(1):2-6.

3. Dog TL. The use of botanicals during pregnancy and lactation. Alternat Ther Health Med 2009;15(1):54-58.

4. Matthews A, Dowswell T, Haas DM, Doyle M, O'Mathuna DP. Interventions for nausea and vomiting in early pregnancy. Cochrane Database Syst Rev 2010;9:CD007575. https://doi. org/10.1002\%2F14651858.CD007575.pub4
5. Bercaw J, Maheshwari B, Sangi-Haghpeykar H. The use during pregnancy of prescription, over-thecounter, and alternative medications among Hispanic women. Birth 2010;37(3):211-218. https://doi. org/10.1111/j.1523-536x.2010.00408.x

6. Lewis JA. The use of herbs by California midwives. Am J Matern Child Nurs 2011;36(4):268. https:// doi.org/10.1111/j.1552-6909.2010.01193.x

7. Lepik K. Safety of herbal medications in pregnancy. Can Pharm J 1997;130(3):29-33.

8. Ernst E. Risk associated with complementary therapies. In: Dukes MNG, Aronson JK, eds. Side-Effects of Drugs. Amsterdam: Elsevier, 2000: 1649

9. Talt PA, Vora A, James S, Fitzgerald DI, Pester BA. Severe congenital lead poisoning in a preterm infant due to a herbal remedy. Med J Aust 2002:177(4):193-195. https://doi.org/10.5694/j.1326-5377.2002. tb04731.x

10. Centers for Disease Control and Prevention. Lead poisoning in pregnant women who used Ayurvedic medications from India - New York City, 2011-2012. Morb Mortal Wkly Rep 2012;61(33):641-646.

11. Wang CC, Li L, Tang LY, Leung PC. Safety evaluation of commonly used Chinese herbal medicines during pregnancy in mice. Hum Reprod 2012;27(8):2448-2456. https://doi.org/10.1093/humrep/des180

12. Chuang CH, Doyle P, Wang JD, Chang PJ, Lai JN, Chen PC. Herbal medicines used during the first trimester and major congenital malformations: An analysis of data from a pregnancy cohort study. Drug Saf 2006;29(6):537-548. https://doi.org/10.2165/00002018-200629060-00006

13. Mabina MH, Pitsoe SB, Moodley J. The effect of traditional herbal medicine on pregnancy outcome: The King Edward VIII Hospital experience. S Afr Med J 1997;87(10):1008-1010.

14. Varga CA, Veale JH. Isihlambezo: Utilization patterns and potential health effects of pregnancyrelated traditional herbal medicine. Soc Sci Med 1997;44(7):911-924. https://doi.org/10.1016/s0277 9536(96)00104-9

15. Naidu M. Understanding African indigenous approaches to reproductive health: Beliefs around traditional medicine. Ethno Med 2014;8(2):147-156. https://doi.org/10.1080/09735070.2014.11917629

16. Mitri F, Hofmeyr GJ, van Gelderen CJ. Meconium during labour: Self-medication and other associations. S Afr Med J 1987;71(7):431-433

17. Holst L, Wright D, Haavik S, Nordeng H. Safety and efficacy of herbal remedies in obstetrics: Review and clinical implications. Midwifery 2011;27(1):80-86. https://doi.org/10.1016/j.midw.2009.05.010

18. Rahman AA, Sulaiman SA, Ahmad Z, Wan Daud WN, Hamid AM. Prevalence and pattern of use of herbal medicines during pregnancy in Tumpat district, Kelantan. Malays J Med Sci 2008;15(3):40-48.

19. Sooi LK, Keng SL. Herbal medicines: Malaysian women's knowledge and practice. Evid Based Complement Alternat Med 2013;2013(438139):1-10. https://doi.org/10.1155/2013/438139

20. Teoh CS, Aizul MHI, Suriyani WMWF, et al. Herbal ingestion during pregnancy and post-partum period is a cause for concern. Med J Malaysia 2013;68(2):157-160.

21. Bayisa B, Tatiparthi R, Mulisa E. Use of herbal medicine among pregnant women on antenatal care at Nekemte Hospital, Western Ethiopia. Jundishapur J Nat Pharm Prod 2014;9(4):e17368.

22. Chuang C, Chang P, Hsieh W, Tsai Y, Lin S, Chen P. Chinese herbal medicine use in Taiwan during pregnancy and the postpartum period: A population-based cohort study. Int J Nurs Stud 2009;46(6):787-795. https://doi.org/10.1016/j.jinurstu.2008.12.01

23. Moussally K, Berard A. Exposure to herbal products during pregnancy and risk of preterm birth Eur J Obstet Gynecol Reprod Biol 2010;150(1):107-108. https://doi.org/10.1016/j.ejogrb.2010.02.001

24. Forster DA, Denning A, Wills G, Bolger M, McCarthy E. Herbal medicine use during pregnancy in a group of Australian wo

25. Fakeye TO, Adisa R, Musa IE. Attitude and use of herbal medicines among pregnant women in Nigeria. BMC Complement Altern Med 2009;9(53):1-7. https://doi.org/10.1186\%2F1472-6882-9-53

26. National Department of Health. The 2015 National Antenatal Sentinel HIV \& Syphilis Survey, South Africa. Pretoria: National Department of Health, 2017:1-75.

Accepted 23 November 2020 